\title{
Numerical investigation and modelling of the venous injection of sclerosant foam
}

\author{
K. C. Wong ${ }^{1} \quad$ S. W. Armfield ${ }^{2} \quad$ N. Williamson ${ }^{3}$
}

(Received 26 February 2019; revised 14 October 2019)

\begin{abstract}
Sclerosant foam, a mixture of a surfactant liquid and air, is injected directly into varicose veins as a treatment that causes the vein to collapse. This investigation develops a model that will allow the medical specialist to visualise how the sclerosant foam will interact with the blood and behave within the vein. The process is simulated using a multiphase computational fluid dynamics model with the sclerosant foam considered as a two-phase non-Newtonian power law viscosity liquid. The governing multiphase equations are solved using an Eulerian-Eulerian approach coupled with a population balance model to predict the bubble size distribution within the flow field. The computational results demonstrate similar flow characteristics and flow features to an available set of experimental results. The model predicts the mixing layers between the sclerosant foam and the ambient fluid, and the
\end{abstract}

DOI:10.21914/anziamj.v60i0.14099 gives this article, (c) Austral. Mathematical Soc. 2019. Published November 29, 2019, as part of the Proceedings of the 18th Biennial Computational Techniques and Applications Conference. ISSN 1445-8810. (Print two pages per sheet of paper.) Copies of this article must not be made otherwise available on the internet; instead link directly to the DOI for this article. 
sclerosant liquid and the ambient fluid, as well as the sclerosant liquid coverage on the vein wall and the bubble size distribution within the vein. These quantities are of interest to medical specialists allowing them to assess the treatment feasibility and safety before treating the patients.

\section{Contents}

1 Introduction

2 Problem description

C263

3 Results

C266

4 Discussion and conclusion

C276

\section{Introduction}

Varicose veins are abnormally dilated diseased blood vessels that are typically treated using a minimally invasive procedure called sclerotherapy that entails the localised destruction of the endothelium of the target vein with an injected detergent sclerosing solution (sclerosants). Following injection into the target vessel, sclerosants are not only diluted by the blood but are also neutralised by plasma proteins such as albumin [10]. While liquid sclerosants are common, sclerosants are also are routinely administered as foam whereby liquid sclerosants are pre-mixed at set ratios with room-air to limit the mixing of sclerosant liquid with blood. As well as limiting dilution and deactivation, foam sclerotherapy also allows for increased contact of the sclerosant liquid with the endothelial wall, providing increased treatment efficiency [3]. However, foam sclerotherapy introduces new complications to the procedure, the most serious being the risk of venous gas embolism, whereby a gas bubble 
occludes small blood vessels in the brain [4]. Hence, accurate computational simulations of the flow behaviour of sclerosant foam would be tremendously useful in enhancing the safety of this medical procedure.

Sclerosant foam was previously modelled as a bulk non-Newtonian power law viscosity fluid [11]. However, that simulation was unable to accurately predict the sclerosant liquid coverage of the vein wall or the bubble size distribution in the foam. In the current study, the sclerosant foam is modeled as a two-phase non-Newtonian power law viscosity fluid, which is shown to be more accurate than the bulk non-Newtonian fluid model. The sclerosant liquid used in this study is sodium tetradecyl sulphate (STS). The two-phase sclerosant foam model is then implemented in the Eulerian-Eulerian multiphase model coupled with the population balance model (PBM) [9] to predict the sclerosant foam spreading and the bubble size distributions within the sclerosant foam in the saphenofemoral junction (SFJ) [7, 1]. Fluid dynamics software package ANSYS-FLUENT V15.0 [5] is used to setup and solve the computational model. The results of such a simulation enable medical specialists to evaluate the risks and effectiveness of the treatment prior to injection of sclerosant foam into the patient.

\section{Problem description}

The geometry of the model is based on the SFJ, shown in Figure 1 with the inlet and outlet boundaries labelled. The conduits are initially filled with normal saline, the primary phase fluid, with sclerosant foam, modelled as a dual secondary phase, injected at the needle position, labelled in Figure 1 as the inlet.

The Eulerian-Eulerian multiphase model treats the phases as interpenetrating continua with each phase obtained by solving a set of conservation equations. The coupling of these conservation equations in this computational study is accomplished by sharing the same pressure and interphase exchange for the 


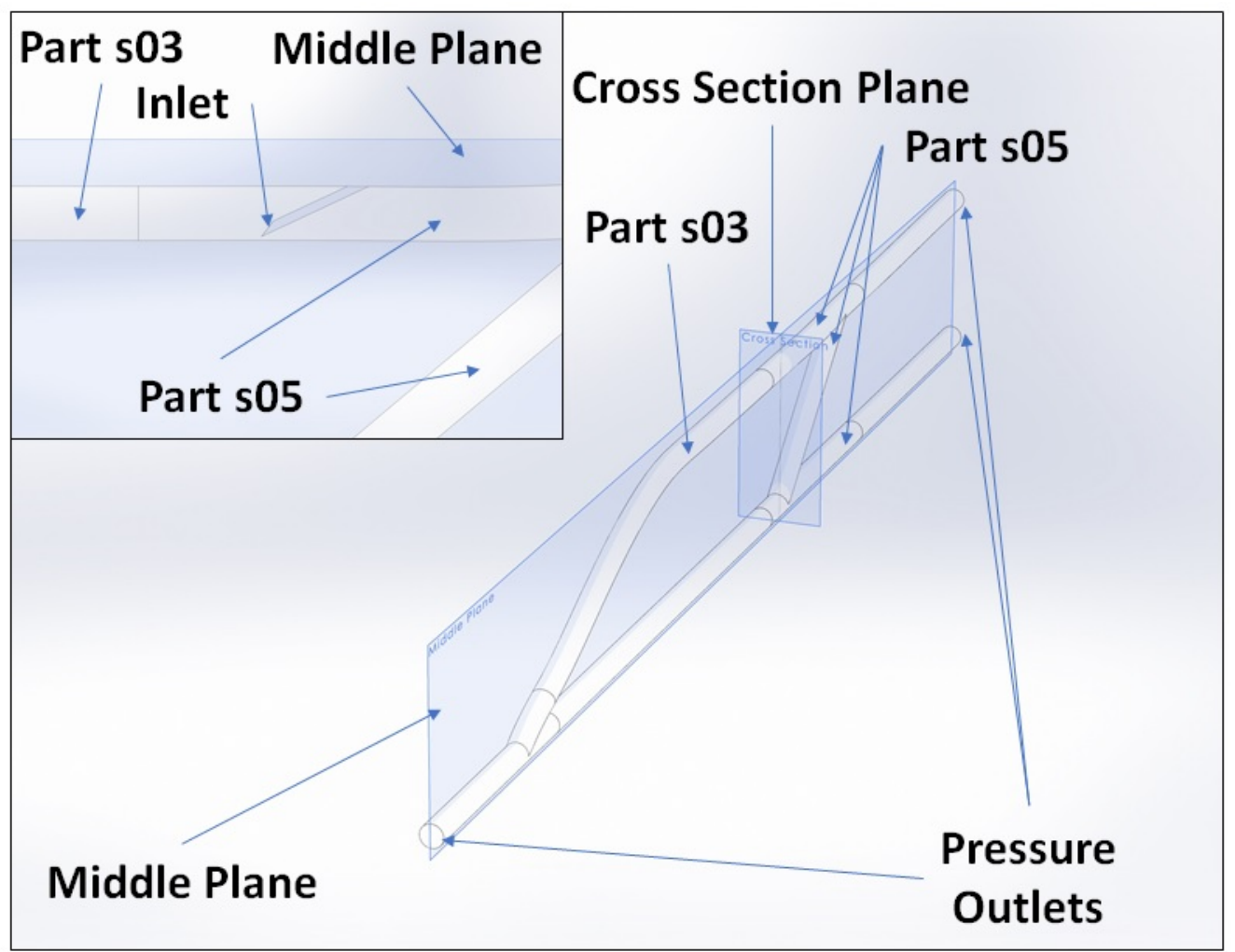

Figure 1: The saphenofemoral junction ( $\mathrm{SFJ}$ ) is located in the thigh of the human body. The simplified SFJ geometry and its boundary zones (inlet are presented for the current numerical model with the inset (middle plane which cuts through parts s03 and s05) showing the inlet. 
drag coefficient. The Eulerian-Eulerian multiphase model governing equations for the conservation of mass (1), momentum (2), and volume fraction (3) are

$$
\begin{aligned}
& \frac{\partial}{\partial t}\left(\alpha_{q} \rho_{q}\right)+\nabla \cdot\left(\alpha_{q} \rho_{q} \mathbf{u}_{q}\right)=0, \\
& \frac{\partial}{\partial t}\left(\alpha_{q} \rho_{q} \mathbf{u}_{q}\right)+\mathbf{u}_{q} \cdot\left(\alpha_{q} \rho_{q} \nabla \mathbf{u}_{q}\right)=-\alpha_{q} \nabla p \\
& +\nabla \cdot\left\{\alpha_{q} \mu_{q, e f f}\left[\nabla \mathbf{u}_{q}+\left(\nabla \mathbf{u}_{q}\right)^{\top}\right]\right\}+\alpha_{q} \rho_{q} \mathbf{g}+\mathbf{F}_{D, q}, \\
& \frac{\partial \alpha_{q}}{\partial t}+\nabla \cdot\left(\alpha_{q} \mathbf{u}_{q}\right)=0,
\end{aligned}
$$

where the subscript $q$ denotes the phase $(q=1$ for normal saline or $q=2$ for STS liquid and air), and $\alpha_{\mathrm{q}}, \rho_{\mathrm{q}}$ and $\mu_{\mathrm{q}}$ denote volume fraction, density and effective viscosity, respectively. The velocity vector is $\mathbf{U}_{\mathbf{q}}$ with gradient $\nabla \mathbf{U}_{\mathrm{q}}$, $p$ is the pressure and $\mathbf{F}_{D, q}$ is the drag force. The normal saline is a Newtonian fluid with effective viscosity

$$
\mu_{1, \text { eff }}=\mu_{1, \text { mol }}+\mu_{1, \mathrm{t}}
$$

where $\mu_{1, \mathrm{~mol}}=1.07 \mathrm{mPas}$ is the saline molecular dynamic viscosity and $\mu_{\mathrm{q}, \mathrm{t}}$ for $\mathrm{q}=1,2$ is the turbulence viscosity obtained via the standard $\mathrm{k}-\epsilon$ turbulence model $[8,6]$. As demonstrated in previous experimental work [13], the sclerosant foam behaves as a non-Newtonian shear thinning fluid and so the effective viscosity for the secondary phases (scelrosant liquid and air) is

$$
\mu_{2, \mathrm{eff}}=\mathrm{K} \dot{\gamma}^{\mathrm{c}-1}+\mu_{2, \mathrm{t}} .
$$

The constant $\mathrm{K}\left(\mathrm{Ns}^{\mathrm{c}} / \mathrm{m}^{2}\right)$ is the consistency index, $\mathrm{c}$ is the flow behaviour index (dimensionless), and $\dot{\gamma}$ is the fluid shear rate. Here we set $\mathrm{K}=1.4257 \mathrm{Ns}^{0.02} / \mathrm{m}^{2}$ and $\mathrm{c}=0.02[13]$.

In this model the sclerosant foam is treated as a two-phase non-Newtonian power law viscosity fluid comprised of sclerosant liquid and air, with the same mixture density, effective viscosity and velocity for both sclerosant liquid and air. The volume fraction for the air is $\alpha_{\text {air }}=0.8$ and the volume fraction for the STS liquid is $\alpha_{\text {STS }}=0.2$. 
The Eulerian-Eulerian multiphase model is coupled with the population balance model (PBM) to solve for the flow field and also track the evolution of the bubble size distribution [9]. The PBM equation (6) is a conservation law for the number of bubbles $n(V, t)$ in a system, with transient and convection terms to track the temporal and spatial evolution of a class of bubbles, balanced with exchange between classes due to coalescence and breakup:

$$
\begin{aligned}
\frac{\partial \mathfrak{n}(\mathrm{V}, \mathrm{t})}{\partial \mathrm{t}}+\nabla \cdot\left[\mathbf{u}_{\mathrm{air}} \mathfrak{n}(\mathrm{V}, \mathrm{t})\right]= & \mathrm{B}_{\mathrm{Co}}(\mathrm{V}, \mathrm{t})-\mathrm{D}_{\mathrm{Co}}(\mathrm{V}, \mathrm{t}) \\
& +\mathrm{B}_{\mathrm{Br}}(\mathrm{V}, \mathrm{t})-\mathrm{D}_{\mathrm{Br}}(\mathrm{V}, \mathrm{t}),
\end{aligned}
$$

where $\mathbf{U}_{\text {air }}$ is the velocity vector for the air phase and $\mathbf{V}$ is the bubble volume. The Kernel terms $B_{\mathrm{Co}}(V, t), D_{\mathrm{Co}}(V, t), B_{\mathrm{Br}}(V, t)$ and $\mathrm{D}_{\mathrm{Br}}(\mathrm{V}, \mathrm{t})$ approximate bubble coalescence birth rate, coalescence death rate, breakage birth rate and breakage death rate, respectively. The kernel model used in this study is based on the Luo coalescence and breakup kernels [5]. We solve the PBM with a discrete method [5].

The semi-implicit method for pressure-linked equations (SIMPLE) was chosen for the pressure-velocity coupling scheme and the equations were integrated in time using a first order explicit approach.

\section{Results}

In the current study, validation tests were conducted with different mesh sizes, time step sizes, bubble groups and discretisation schemes. Results were obtained with average mesh sizes of $2.0 \times 10^{-4} \mathrm{~m}, 1.0 \times 10^{-4} \mathrm{~m}$ and $5.0 \times 10^{-5} \mathrm{~m}$ where the vein diameter is $3.0 \times 10^{-3} \mathrm{~m}$, and time steps $9.5 \times 10^{-5} \mathrm{~s}, 1.9 \times 10^{-4} \mathrm{~s}$ and $3.8 \times 10^{-4} \mathrm{~s}$. The bubbles were grouped into 7, 14 and 21 bins, with the bubble sizes ranging from $15 \mu \mathrm{m}$ to $210 \mu \mathrm{m}$. For the advection term, both the first order upwind and the second order central discretisation schemes were tested. All these combinations provided approximately the same results. For the results present here we use the coarsest mesh size $2.0 \times 10^{-4} \mathrm{~m}$ and 
coarsest time step $3.8 \times 10^{-4}$ s, with 14 bubble bins and the first order upwind discretisation.

Figure 2 shows experimental results at $t=1 \mathrm{~s}$ and $t=4 \mathrm{~s}$ after initiation of the foam injection [12]. For comparison with these experimental results, Figures 3 and 4 show numerical volume fraction distributions for the normal saline, STS liquid and air at times $t=1 \mathrm{~s}$ and $t=4 \mathrm{~s}$, respectively. The numerical results provide reasonable predictions of the experimental foam spread and structure. Figure 3 shows a buffer layer consisting of a mixture of normal saline and sTs liquid between the normal saline and the foam. Figure 5 shows the $t=4 \mathrm{~s}$ bubble group size distribution on the middle plane of the geometry and the air phase volume fraction distribution. The region near the inlet (indicated in Figure 5 by the uncoloured needle-shaped region) is dominated by small bubbles which are seen to form a plume. Away from the inlet, coalescence of small bubbles leads to large bubbles dominating.

Figures 6 and 7 show numerical volume fraction distributions of the phases on the radial cross section of the vein at the inlet (indicated by the uncoloured region in the cross section) at times $t=1 \mathrm{~s}$ and $t=4 \mathrm{~s}$, respectively. As the flow progresses from $t=1 \mathrm{~s}$ to $t=4 \mathrm{~s}$ the foam sclerosant displaces the normal saline towards the bottom of the conduit, and ultimately completely out of this section of the domain. Within the foam the air accumulates towards the upper region as a result of buoyancy forces. Similarly to Figure 3, Figure 6 shows a buffer layer of normal saline and sTs liquid between the normal saline and the foam.

Figures 8 and 9 show numerical results for the small and large bubble concentrations on the radial cross section of the vein at the inlet at times $t=1 \mathrm{~s}$ and $t=4 \mathrm{~s}$, respectively. Similarly to Figure 5 , we again observe that the smaller bubbles dominate near the inlet plume region, while the larger bubbles dominate far from the inlet region due to coalescence of small bubbles. 

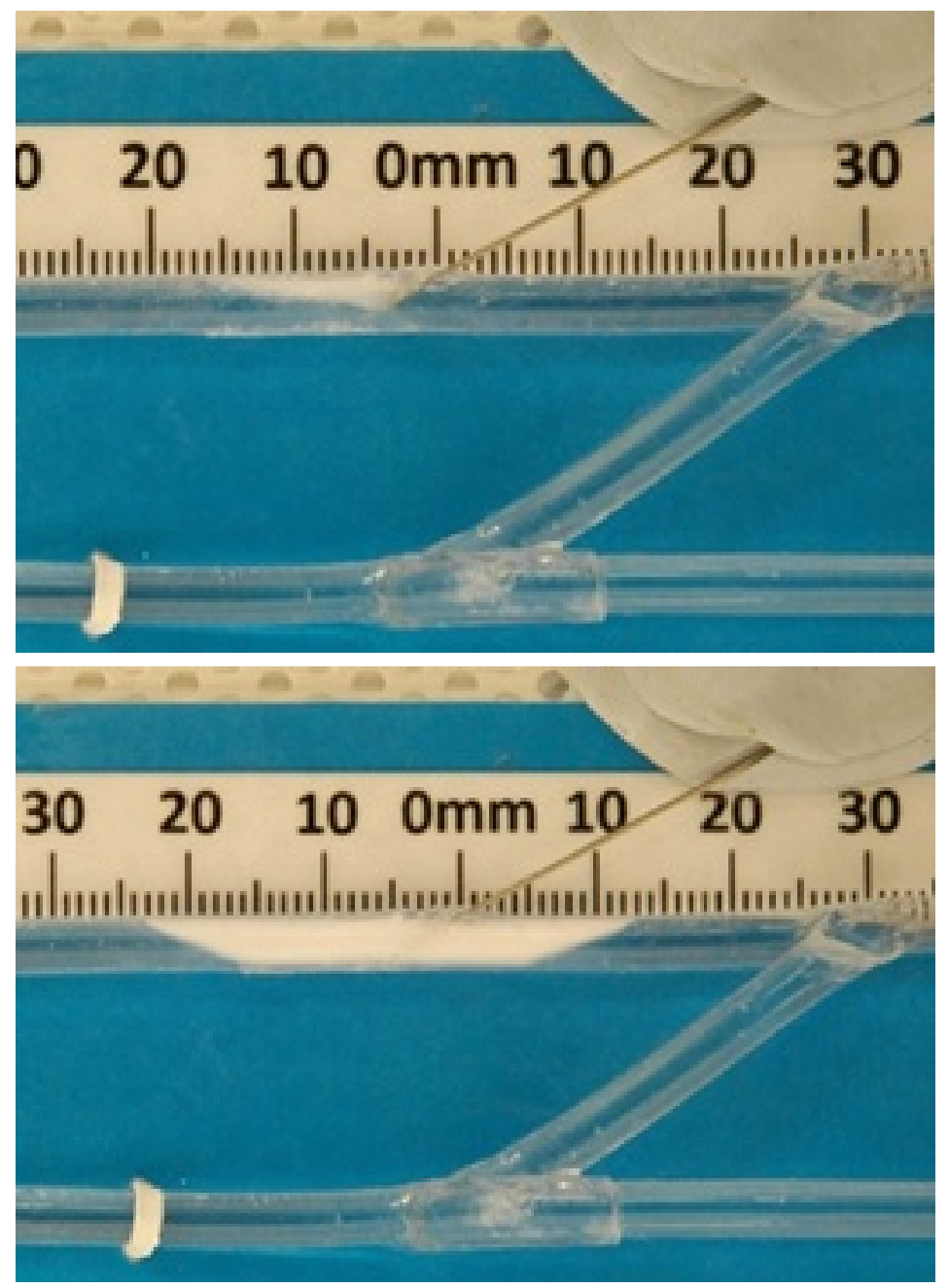

Figure 2: Experimental results at (top) $t=1 \mathrm{~s}$ and (bottom) $t=4 \mathrm{~s}$, with normal saline clear and foam white. 


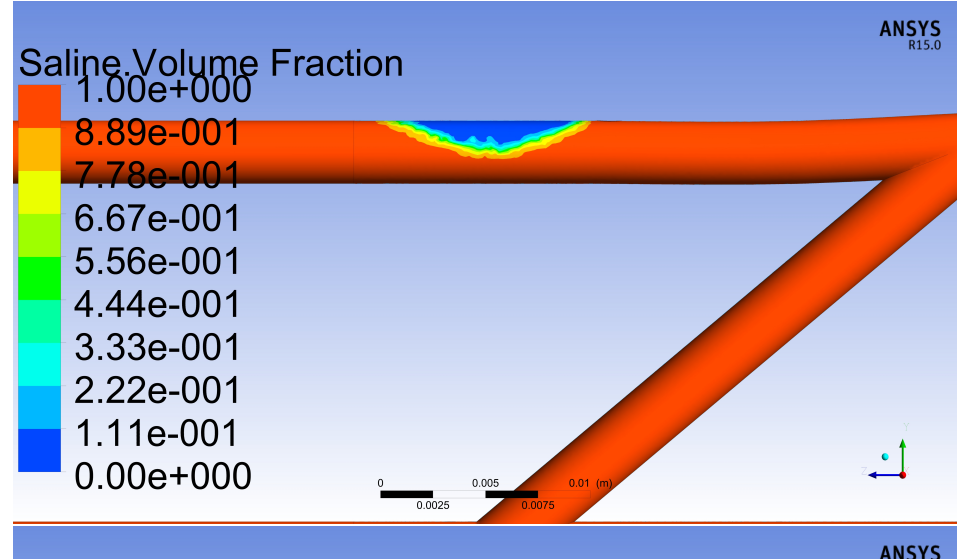

Stsfoam. Volume Fraction

ANSYS

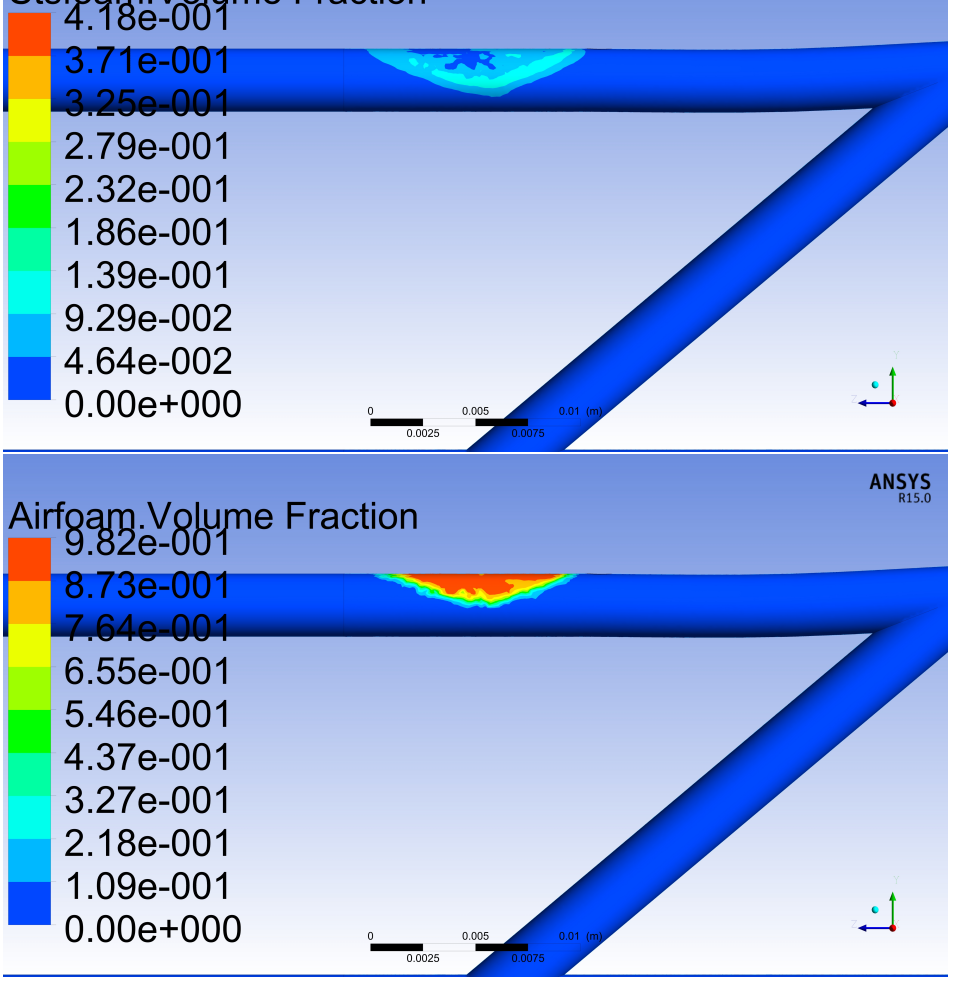

Figure 3: Numerical results at $t=1 \mathrm{~s}$ for volume fraction distributions of: (top) normal saline; (middle) sts liquid; and (bottom) air. 


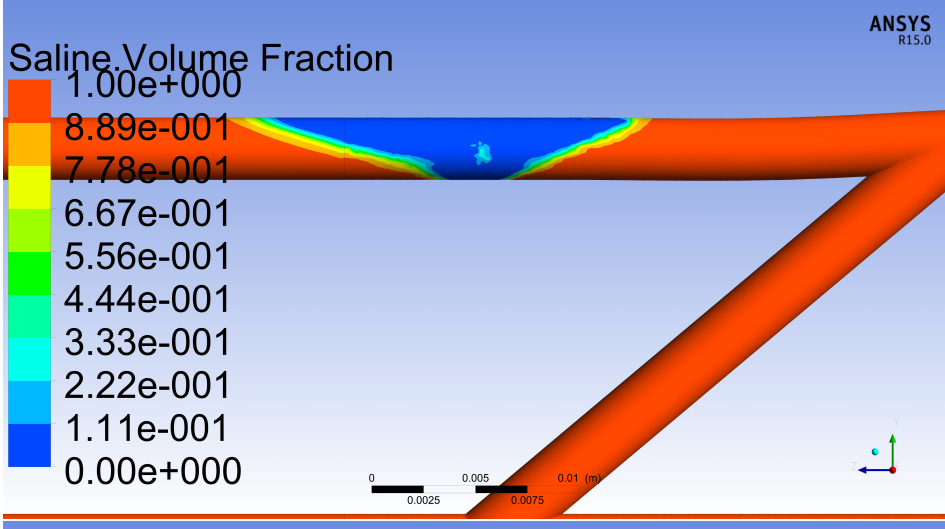

Stsfoam. Volume Fraction

ANSYS

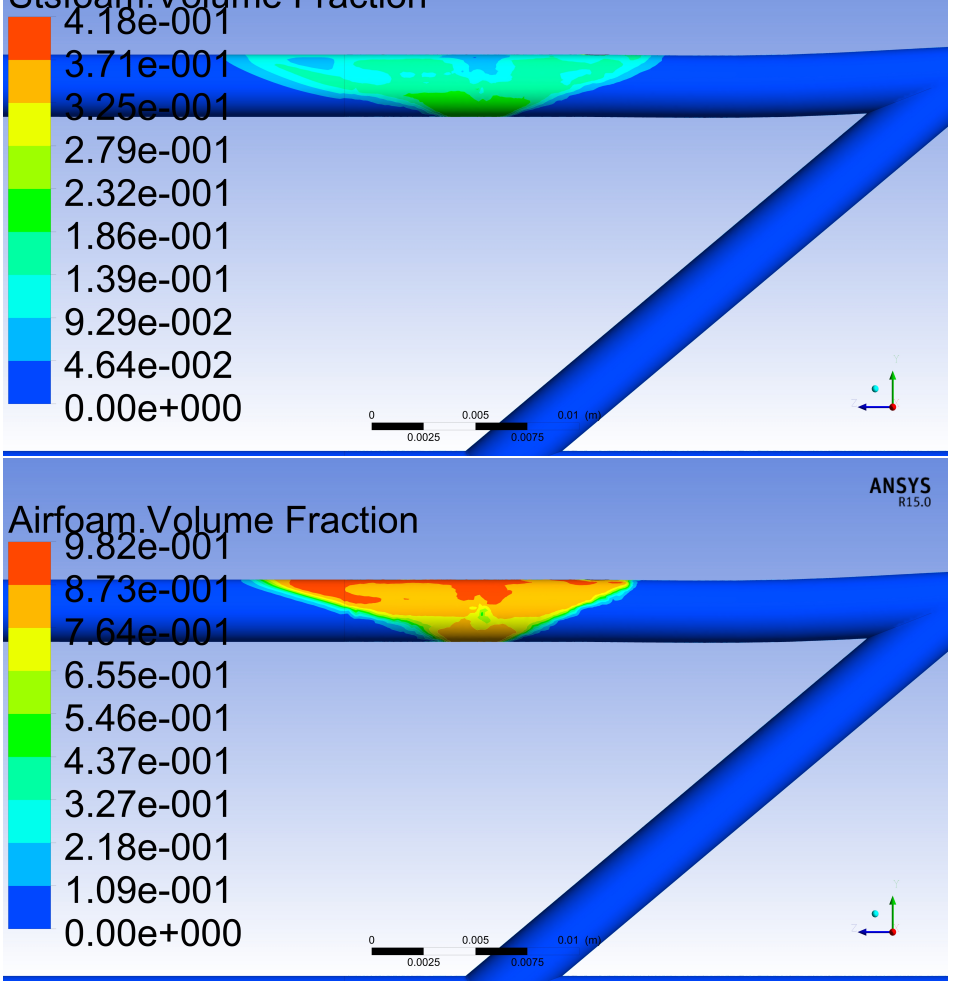

Figure 4: Numerical results at $t=4 \mathrm{~s}$ for volume fraction distributions of: (top) normal saline; (middle) sts liquid; and (bottom) air. 

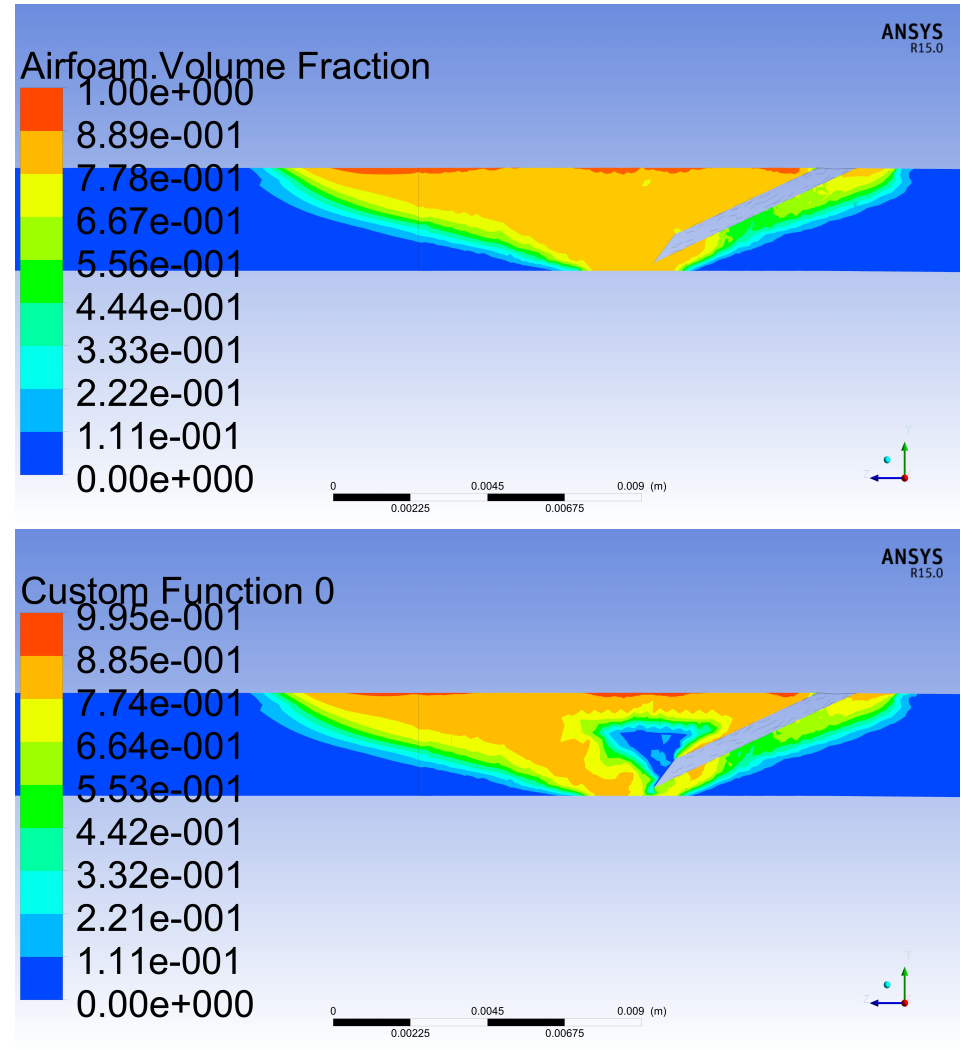

Custom Function 12

ANSYS

2.17e-001

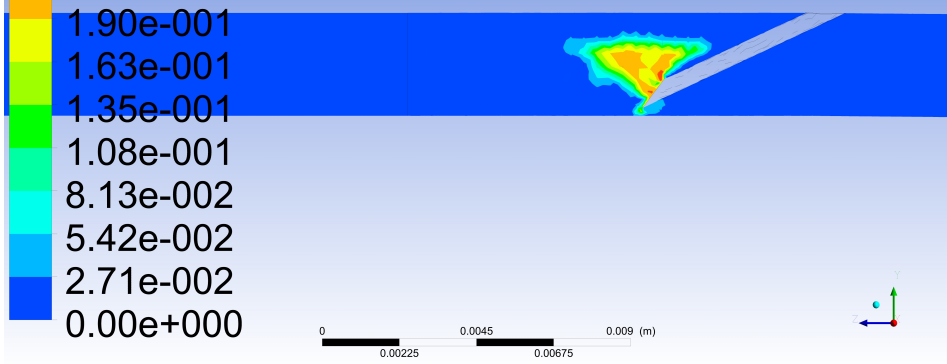

Figure 5: Numerical results at $\mathrm{t}=4 \mathrm{~s}$ for: (top) air volume fraction distribution; (middle) distribution of large bubbles of size $210 \mu \mathrm{m}$ (bubble bin 0); and (bottom) distribution of small bubbles of size $30 \mu \mathrm{m}$ (bubble bin 12). 


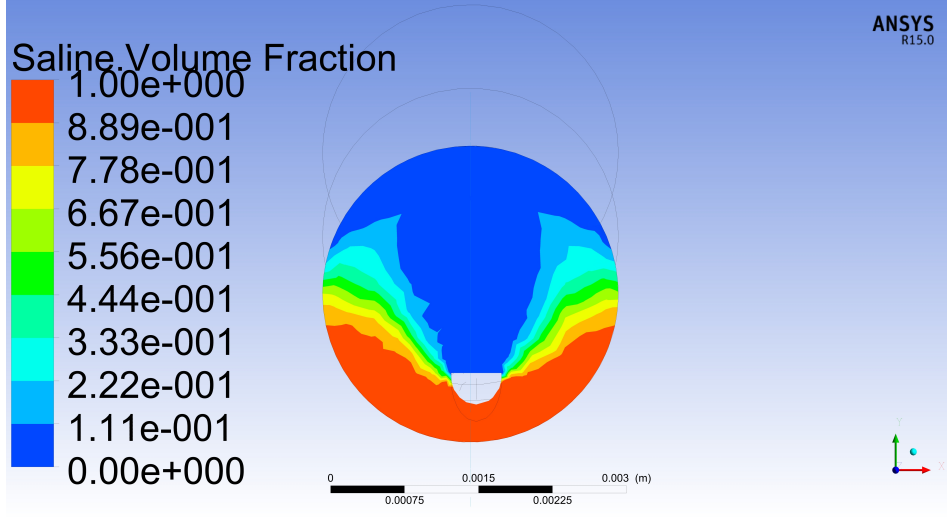

Stsfoam. Volume Fraction

ANSYS

3.71e-001

$3.25 \mathrm{e}-001$

$2.79 \mathrm{e}-001$

2.32e-001

$1.86 \mathrm{e}-001$

$1.39 \mathrm{e}-001$

$9.29 \mathrm{e}-002$

$4.64 \mathrm{e}-002$

$0.00 \mathrm{e}+000$
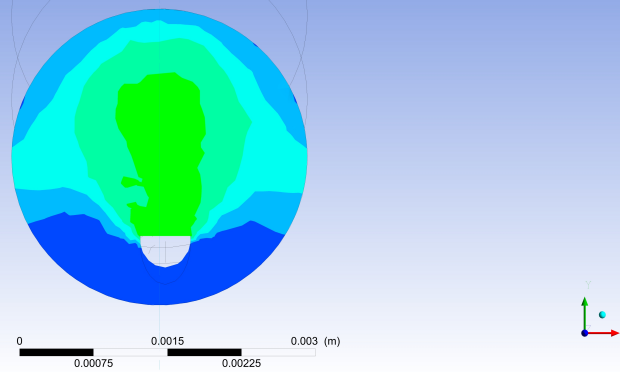

Airfoam.Volume Fraction

ANSYS

8.89e-001

7.78e-001

$6.67 \mathrm{e}-001$

$5.56 \mathrm{e}-001$

$4.44 \mathrm{e}-001$

3.33e-001

$2.22 \mathrm{e}-001$

$1.11 \mathrm{e}-001$

$0.00 e+000$

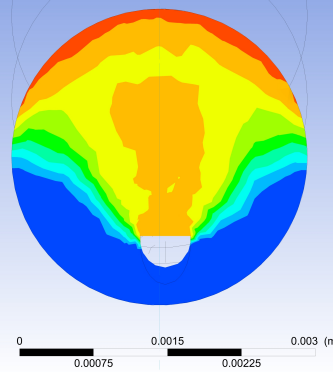

$\stackrel{\circ}{\longrightarrow}$

Figure 6: Numerical results of the radial cross section at the inlet at time $\mathrm{t}=1 \mathrm{~s}$ for volume fraction distributions of: (top) normal saline; (middle) STS liquid; and (bottom) air. 


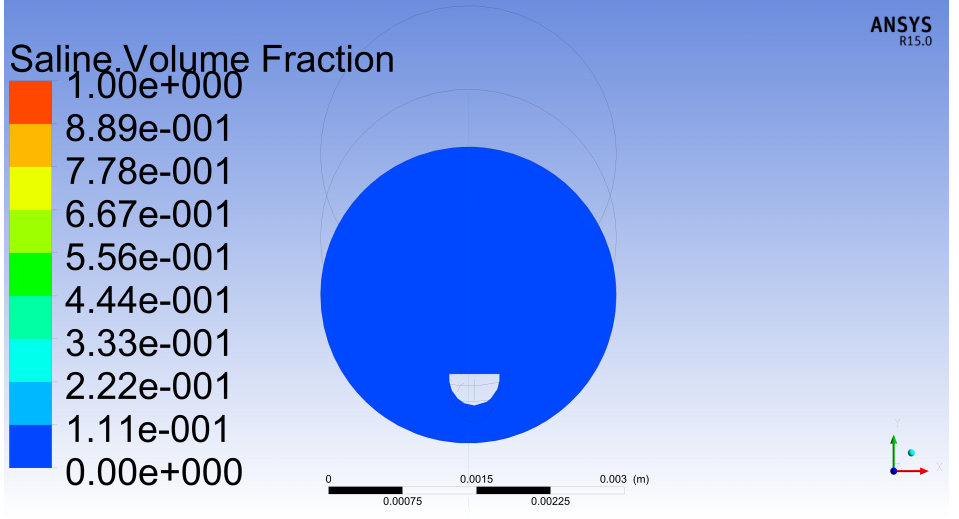

Stsfoam.Volume Fraction

ANSYS

3.71e-001

3. $25 \mathrm{e}-001$

2.79e-001

2.32e-001

$1.86 \mathrm{e}-001$

$1.39 \mathrm{e}-001$

$9.29 \mathrm{e}-002$

$4.64 \mathrm{e}-002$

$0.00 \mathrm{e}+000$

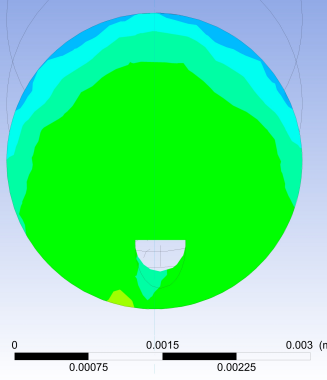

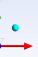

Airfoam. Volume Fraction

ANSYS

8.89e-001

7.78e-001

6.67e-001

5.56e-001

4.44e-001

3.33e-001

2.22e-001

$1.11 \mathrm{e}-001$

$0.00 e+000$

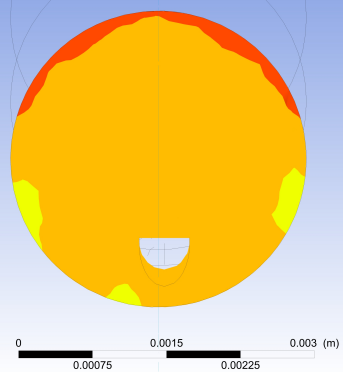

$\stackrel{\circ}{\circ}$

Figure 7: Numerical results of the radial cross section at the inlet at time $\mathrm{t}=4 \mathrm{~s}$ for volume fraction distributions of: (top) normal saline; (middle) STS liquid; and (bottom) air. 

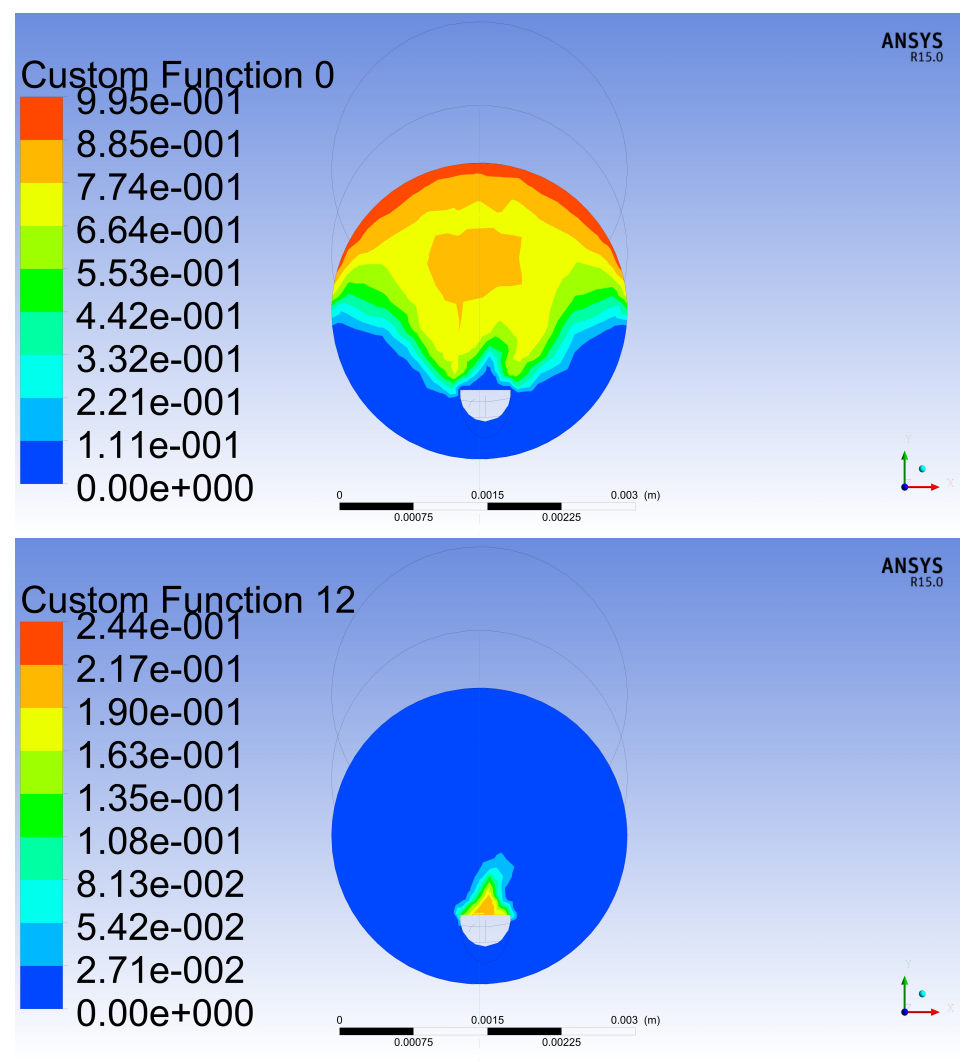

Figure 8: Bubble size distributions on a radial cross section at the inlet at $\mathrm{t}=1 \mathrm{~s}$ for (top) large bubbles of size $210 \mu \mathrm{m}$ (bubble bin 0 ) and (bottom) small bubbles of size $30 \mu \mathrm{m}$ (bubble bin 12). 

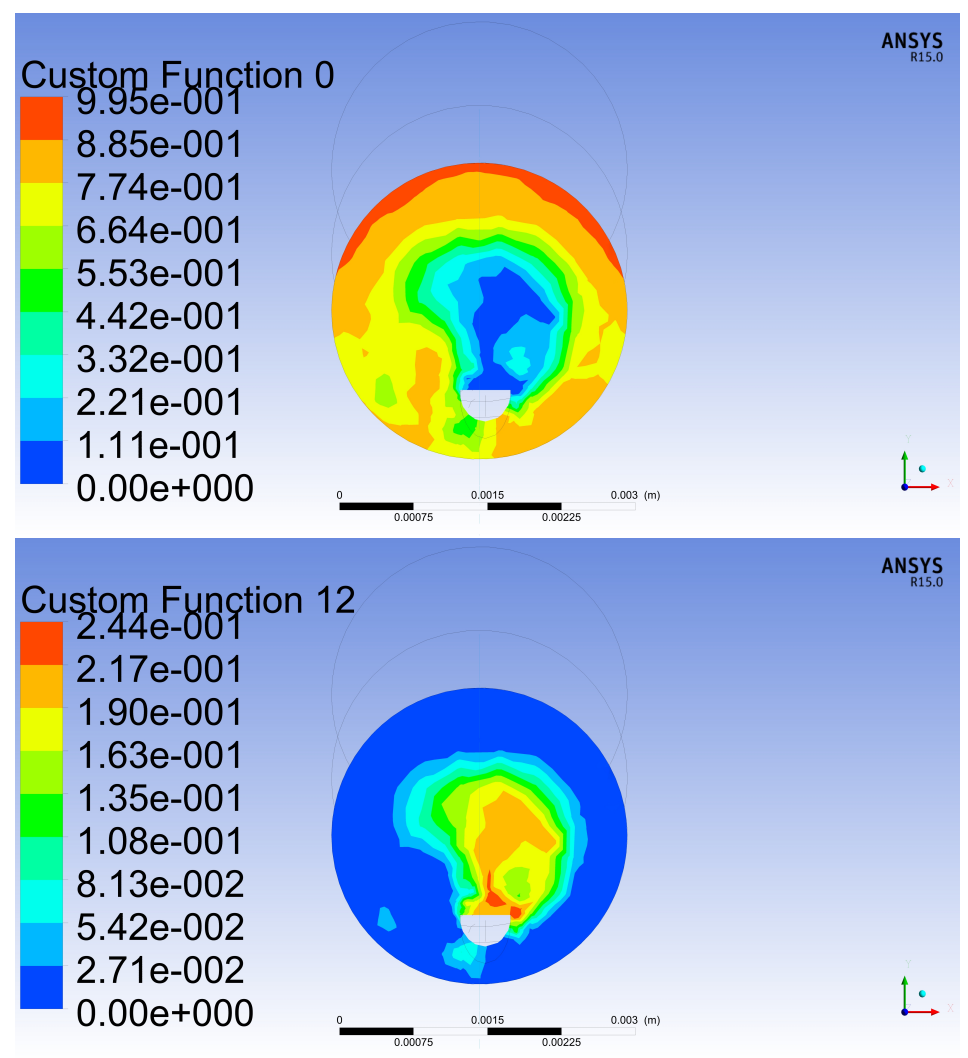

Figure 9: Bubble size distributions on a radial cross section at the inlet at $\mathrm{t}=4 \mathrm{~s}$ for (top) large bubbles of size $210 \mu \mathrm{m}$ (bubble bin 0 ) and (bottom) small bubbles of size $30 \mu \mathrm{m}$ (bubble bin 12). 


\section{Discussion and conclusion}

The model used here is shown to provide reasonable accuracy in its prediction of the overall spread and structure of the sclerosant foam. In particular it accurately predicts the adherence of the foam to the tube wall, as shown in Figure 3, which was not predicted previously [11]. This result enables the medical specialist to visualise how the sclerosant foam spreads within the SFJ, and to quantify the STS liquid coverage on the vein wall.

The PBM allows the computational model to predict the bubble size distribution. As shown in Figures 5, 8 and 9, the generation of larger bubbles is unavoidable as smaller bubbles coalesce as the foam plume spreads from the inlet in both the axial and vertical directions. Accurate predictions of the occurrence and location of larger bubbles is an invaluable aid in assessing the risk of embolism.

Figures 3 and 6 show that the three phases mix with each other, forming buffer layers between the phases. The buffer layer between the normal saline and liquid sclerosant, associated with dilution of the sclerosant, has also been observed experimentally [2], and reduces the effectiveness of the treatment [10].

The presented computational fluid dynamics study successfully integrates three physical models (Eulerian-Eulerian multiphase, non-Newtonian power law viscosity and population balance model) to analyse sclerosant foam flow, providing reasonable agreement with experimental results. However, there is room for further development. The geometry considered here is idealised; the vein wall is inelastic and the pulsatile blood flow is approximated by non-flowing normal saline fluid. A more realistic model would allow for elastic deforming veins, include bicuspid valves and replace the normal saline with blood. Additionally, other sclerosant fluids, such as Polidocanol, and a greater range of sclerosant concentrations and liquid air fractions should be tested. 


\section{References}

[1] S. Ali Mirjalili, J. C. Muirhead, and M. D. Stringer. "Redefining the surface anatomy of the saphenofemoral junction in vivo". In: Clin. Anat. 27.6 (2014), pp. 915-919. DOI: 10.1002/ca.22386 (cit. on p. C263).

[2] E. Cameron, T. Chen, D. E. Connor, M. Behnia, and K. Parsi. "Sclerosant foam structure is strongly influenced by liquid air fraction". In: Eur. J. Vasc. Endo. Surg. 46 (2013), pp. 488-494. DOI: 10.1016/j.ejvs.2013.07.013 (cit. on p. C276).

[3] P. Coleridge-Smith. "Saphenous ablation: Sclerosant or sclerofoam?" In: Semin. Vasc. Surg. 18 (2005), pp. 19-24. DOI: 10.1053/j. semvascsurg. 2004.12.007 (cit. on p. C262).

[4] J.-J. Guex. "Complications and side-effects of foam sclerotherapy". In: Phlebology 24 (2009), pp. 270-274. DOI: 10.1258/phleb.2009.009049 (cit. on p. C263).

[5] ANSYS. Inc. ANSYS FLUENT 12.0 population balance module manual. ANSYS, 2010. URL: https://www.afs.enea.it/project/neptunius/ docs/fluent/html/popbal/main_pre.htm (cit. on pp. C263, C266).

[6] F. Ren, N. A. Noda, T. Ueda, Y. Sano, Y. Takase, T. Umekage, Y. Yonezawa, and H. Tanaka. "CFD-PBM coupled simulation of a nanobubble generator with honeycomb structure". In: vol. 372. IOP Conference Series: Materials Science and Engineering. June 2018, p. 012012. DOI: 10.1088/1757-899X/372/1/012012 (cit. on p. C265).

[7] P. Souroullas, R. Barnes, G. Smith, S. Nandhra, D. Carradice, and I. Chetter. "The classic saphenofemoral junction and its anatomical variations". In: Phlebology 32.3 (2017), pp. 172-178. DOI: 10.1177/0268355516635960 (cit. on p. C263).

[8] A. H. Syed, M. Boulet, T. Melchiori, and J. M. Lavoie. "CFD simulations of an air-water bubble column: Effect of Luo coalescence parameter and breakup kernels". In: Front. Chem. 5.68 (2017), pp. 1-16. DOI: 10.3389/f chem.2017.00068 (cit. on p. C265). 
[9] T. Wang and J. Wang. "Numerical simulation of gas-liquid mass transfer in bubble column with a CFD-PBM coupled model". In: Chem. Eng. Sci. 62 (2007), pp. 7107-7118. DOI: 10.1016/j.ces.2007.08.033 (cit. on pp. C263, C266).

[10] M. R. Watkins. "Deactivation of sodium tetradecyl sulphate injection by blood proteins". In: Euro. J. Vasc. Endo. Surg. 41.4 (2011), pp. 521-525. DOI: 10.1016/j.ejvs.2010.12.012 (cit. on pp. C262, C276).

[11] K. C. Wong, T. Chen, D. E. Connor, M. Behnia, and K. Parsi. "Computational fluid dynamics of liquid and foam sclerosant injection in a vein model". In: Appl. Mech. Mater. 553 (2014), pp. 293-298. DOI: 10.4028/www. scientific.net/AMM. 553.293 (cit. on pp. C263, C276).

[12] K. Wong. "Experimental and numerical investigation and modelling of sclerosant foam". PhD thesis. University of Sydney, 2018 (cit. on p. C267).

[13] K. Wong, T. Chen, D. E. Connor, M. Behnia, and K. Parsi. "Basic physiochemical and rheological properties of detergent sclerosants". In: Phlebology 30.5 (2015), pp. 339-349. DOI: 10.1177/0268355514529271 (cit. on p. C265).

\section{Author addresses}

1. K. C. Wong, School of Aerospace, Mechanical and Mechatronic Engineering, University of Sydney, New South Wales 2006, Australia. mailto:kwon6774@uni.sydney.edu.au

2. S. W. Armfield, School of Aerospace, Mechanical and Mechatronic Engineering, University of Sydney, New South Wales 2006, Australia.

3. N. Williamson, School of Aerospace, Mechanical and Mechatronic Engineering, University of Sydney, New South Wales 2006, Australia. 